\title{
Imaging Immunological Processes from Blood to Brain in ALS
}

\author{
Sandra Amor ${ }^{1}$, Erik Nutma ${ }^{1}$, Manuel Marzin ${ }^{1}$, and Fabiola Puentes ${ }^{2}$ \\ ${ }^{1}$ VU medisch centrum School of Medical Sciences \\ ${ }^{2}$ Barts and The London School of Medicine and Dentistry Blizard Institute
}

June 10, 2021

\begin{abstract}
Neuropathology studies of amyotrophic lateral sclerosis (ALS) and animal models of ALS reveal a strong association between aberrant protein accumulation and motor neuron damage and activated microglia and astrocytes, the resident CNS innate immune cells. While the role of neuroinflammation in the pathology of ALS is unclear imaging studies support the idea that innate immune activation occurs early disease in both humans and rodent models of ALS. In addition to innate immunity, emerging studies also reveal the presence of peripheral monocytes, macrophages, and lymphocytes in the CNS as well as at the neuromuscular junction. To better understand the association of neuroinflammation (innate and adaptive) with disease progression paraclinical studies including the use of biomarkers and imaging modalities allow monitoring of immune parameters in the disease process. Such approaches are important for patient stratification, selection, and inclusion in clinical trials, as well as to provide readouts of response to therapy. Here, we discuss the different imaging modalities e.g., MRI, MRS, PET as well as other approaches including biomarkers of inflammation in ALS, aid the understanding of the underlying immune mechanisms associated with motor neuron degeneration in ALS.
\end{abstract}

\section{Hosted file}

ALS Imaging Neuroinflammation.docx available at https://authorea.com/users/336214/articles/ 525704-imaging-immunological-processes-from-blood-to-brain-in-als 